\title{
Patient safety: research methods for a new field
}

\section{J B Battles}

Correspondence to: Dr J B Battles, Center for Quality Improvement and Patient Safety, Agency for Healthcare Research and Quality (AHRQ), 540 Gaither Road, Rockville, MD 20850, USA; jbattles@ahrq.gov
$D{ }^{a}$ tient safety has emerged as a significant public health issue at the beginning of the 21 st century. In the few years since the release of the Institute of Medicine's report "To Err is Human"1 in the United States and "An Organization with a Memory" ${ }^{2}$ in the UK, major research activities have been launched to address issues of patient safety, medical error, and patient injury or harm. This fury of interest in patient safety has raised the question of just what are the appropriate research methods for this new field? Can issues of patient safety be addressed with traditional research methods associated with health services research? Are new methods and approaches required? If new methods and approaches are required, what are they and how can they be applied to patient safety issues and concerns?

This special supplemental issue of Quality and Safety in Health Care begins to answer some of these questions by presenting a series of articles that describe research methods and approaches to identify risks and hazards related to patient safety. Most of the papers were initially prepared for the first United States/United Kingdom Patient Safety Research Methodology Workshop. This workshop was jointly sponsored by the US Agency for Healthcare Research and Quality (AHRQ) and the Patient Safety Research Programme of the UK Department of Health held in September 2002 in Reykjavik, Iceland. The workshop sought to bring together 25 of the researchers at the leading edge of investigation relevant to the improvement of safety in both the US and the UK. So that the material heard by attendees at the workshop can be available to a wider audience, the authors have translated workshop discussion papers into articles for this supplement.

Battles and Lilford provide an overview and conceptual framework for patient safety research. The next group of articles by Lilford, Zhan, and Rozich focus on the use of existing patient records and discharge information to identify risk and hazards. The articles by Kaplan, Tsatsoulis, Johnson, and Henriksen all consider issues related to event reporting systems, while both Carthey and Mackenzie examine approaches for direct observation to detect risks and hazards. Marx introduces the concept of probabilistic risk assessment (PRA) as a proactive method of risk identification, and Nieva explores the measurement of organizational culture within the framework of identification of risks and hazards.

It is hoped that this collection of articles on research methods will be helpful to the readers of QSHC who are planning to conduct patient safety research, as well as to those who must evaluate such research in considering adoption of interventions aimed at eliminating the risks and hazards associated with patient safety.

\section{REFERENCES}

1 Kohn LT, Corrigan JM, Donaldson MS, eds. To err is human building a safer health system. Washington, DC: National Academy Press, 1999.

2 Department of Health. An organization with a memory: a report of an expert group on learning from adverse events in the NHS. London: Department of Health, 2000. 